\title{
In Defense of the LDL Hypothesis
}

\author{
David S. Schade ${ }^{*}$, Lynda Shey², R. Philip Eaton ${ }^{1}$ \\ ${ }^{1}$ Division of Endocrinology and Metabolism, Department of Internal Medicine, School of Medicine, University of New Mexico, \\ Albuquerque, NM, USA \\ ${ }^{2}$ Diabetes Comprehensive Care Center, University of New Mexico Hospital, Albuquerque, NM, USA \\ Email: *dschade@salu d.unm.edu
}

How to cite this paper: Schade, D.S., Shey, L. and Eaton, R.P. (2019) In Defense of the LDL Hypothesis. World Journal of Cardiovascular Diseases, 9, 245-252. https://doi.org/10.4236/wjcd.2019.93022

Received: February 20, 2019

Accepted: March 24, 2019

Published: March 27, 2019

Copyright (c) 2019 by author(s) and Scientific Research Publishing Inc. This work is licensed under the Creative Commons Attribution International License (CC BY 4.0).

http://creativecommons.org/licenses/by/4.0/ (c) (i) Open Access

\begin{abstract}
Both clinical and basic science studies during the last 30 years have emphasized the importance of cholesterol in the pathogenesis of atherosclerosis. There is a direct relationship between the level of circulating cholesterol (as LDLc remnant particles) and the incidence of cardiovascular events. However, this hypothesis has not gone unchallenged, both in social media and the scientific literature. This controversy has major consequences to the health of the public, because atherosclerosis is the number one cause of morbidity and mortality in the Western World. The proponents of the non-importance of atherosclerosis base their argument on certain studies. We have carefully examined these studies in order to address the validity of the challenges. Each of these studies has its own deficiencies as pointed out in this article. Overall, the evidence that cholesterol is not the primary underlying pathogenic factor causing heart attacks and strokes is based on a flawed interpretation of study results. These studies have several serious deficiencies including lost data, inadequate power, excessive drop outs, borderline significance, and lack of a control group. The conclusion that cholesterol is essential in the pathogenesis of atherosclerosis is critical for treating and preventing atherosclerosis and reducing the prevalence of cardiovascular disease.
\end{abstract}

\section{Keywords}

Atherosclerosis, Cholesterol, LDL Cholesterol, Study Design, Statins, Cardiovascular Disease

\section{Introduction}

During the last decade, many studies have been published examining the role of cholesterol in the pathogenesis of cardiovascular disease (CVD). There have been several extensive reviews of the pathogenesis of atherosclerosis [1]. The 
atherosclerotic process is complex, but begins in childhood and continues throughout life [2]. It begins with the entrance of LDL cholesterol into the arterial vessel wall and the activation of the inflammatory process [3]. Macrophages engulf oxidized LDL cholesterol, coalesce into a fatty streak, become surrounded by inflammatory cells and cytokines, and form an unstable atherosclerotic plaque in the vessel wall [4]. The plaque containing cholesterol can then undergo three different fates. First, it can become stabilized with progressive calcification, development of a thick fibrous cap, and a reduction in inflammatory elements. Second, the cholesterol in the plaque can be slowly removed via reverse cholesterol transport, primarily through the activity of high density lipoprotein particles. This "plaque reversal" occurs at an LDL cholesterol concentration of approximately $70 \mathrm{mg} / \mathrm{dl}$ [5]. The third fate is for the plaque to rupture into the artery, with cholesterol and other inflammatory elements activating a thrombotic cascade, eventually resulting in arterial obstruction and a heart attack or stroke. Statins have been shown to not only accelerate the removal of the plaque via a reduction in LDL cholesterol, but also by acting acutely to reduce the inflammation [6]. Thus, statins have pleotropic effects on reducing atherosclerosis.

A large cohort of randomized controlled clinical trials have established the efficacy of statin-induced cholesterol reduction in preventing cardiovascular events [7] [8]. This observation is valid even in individuals at low cardiovascular risk [9]. A consensus in the medical community now accepts the LDLc hypothesis that cholesterol (particularly low density lipoprotein cholesterol, i.e. LDLc) is the primary culprit in the formation of atherosclerotic plaques [10]. However, the history of medicine is replete with examples of established medical therapies that later were proven to be false. Therefore, challenging the status quo is an important function of scientists seeking the truth. To this end, a recent report challenges the importance of cholesterol (LDLc) in the pathogenesis of atherosclerosis [11]. In addition, the internet is replete with warnings concerning the use of statins to lower cholesterol to prevent CVD [12]. Examining the validity of this challenge is also important in order to achieve a scientifically based consensus. There are at least four approaches to respond to a challenge to accepted scientific dogma. First is to examine the validity of the scientific evidence cited by the challenger; second is to assess the weight of the totality of evidence; third is to correlate the pathogenesis of the disease with the therapeutic intervention; and fourth is to evaluate the response to therapy and its reversal of the disease. This article applies the first approach, i.e., to address the scientific validity of the cited evidence purporting to support an alternative to the "LDL hypothesis" that the lower the LDLc, the less the cardiovascular disease.

\section{Study Strengths and Weaknesses}

Table 1 lists the studies that have been cited to support the claim that LDLc lowering (primarily by statins) did not result in a reduction of atherosclerotic 
Table 1. Deficiencies of cited studies suggesting No LDCc lowering benefit.

\begin{tabular}{|c|c|c|c|c|}
\hline $\begin{array}{l}\text { Study name or } \\
\text { Acronym }\end{array}$ & $\begin{array}{c}\text { Study Deficiency } \\
\# 1\end{array}$ & $\begin{array}{c}\text { Study Deficiency } \\
\# 2\end{array}$ & $\begin{array}{c}\text { Study Deficiency } \\
\# 3\end{array}$ & $\begin{array}{c}\text { Comments and } \\
\text { Concerns } \\
\text { (Table 2) }\end{array}$ \\
\hline \#1 St. Francis & $\downarrow$ Stat & Prot Def & $\uparrow$ Drop in/out & yes \\
\hline \#2 IDEAL & $\downarrow \mathrm{CVD}$ & $\downarrow$ Stat & Prot Def & yes \\
\hline \#3 FIELD & LDL Rcpt & $\downarrow$ Stat & CRP? & yes \\
\hline$\# 44 \mathrm{D}$ & CRP? & $\uparrow$ Drop in/out & --- & yes \\
\hline \#5 ASPEN & $\downarrow$ Stat & $\uparrow$ Drop in/out & Prot Def & yes \\
\hline \#6 WHI & CRP? & $\downarrow \mathrm{CVD}$ & $\uparrow$ Side effcs & yes \\
\hline \#7 ILLUMINATE & $\downarrow$ Observ & LDL Rcpt & $\uparrow$ Side effcs & yes \\
\hline \#8 CDRONA & $\downarrow$ Observ & $\downarrow$ Stat & --- & yes \\
\hline \#9SEAS & CRP? & Prot Def & --- & yes \\
\hline \#10 GISSI-HF & Prot Def & CRP? & $\downarrow$ Stat & yes \\
\hline \#11 AURORA & $\downarrow$ Observ & CRP? & --- & yes \\
\hline \#12 SEARCH & CRP? & $\uparrow$ Drop in/out & Prot Def & yes \\
\hline \#13 AIM-HIGH & LDL Rcpt & Prot Def & $\uparrow$ Side effcs & yes \\
\hline \#14 SDHS & CRP? & LDL Rcpt & Prot Def & yes \\
\hline \#15 HPS2-THRIVE & LDL Rcpt & Prot Def & $\uparrow$ Side effcs & yes \\
\hline \#16 ACCELERATE & LDL Rcpt & $\uparrow$ Side effcs & CRP? & yes \\
\hline \#17 HIJ-PROPER & $\downarrow$ Stat & Prot Def & $\downarrow$ Power & yes \\
\hline \#18 EMPATHY & $\downarrow$ Stat & $\uparrow$ Drop in/out & Prot Def & yes \\
\hline
\end{tabular}

$\downarrow$ Obser = Observation time too short. No CRP? = Failure to monitor or normalize the concomitant change in C-reactive protein. LDL Rcpt $=$ Use of an LDLc lowering agent that does not increase hepatic LDL receptors. $\downarrow \mathrm{CVD}=$ Unexpectedly low rate of cardiovascular events. $\downarrow$ Stat $=$ Lack of reaching the significant level of $\mathrm{p}<0.05$. $\uparrow$ Drop in/out $=$ Excessive dropins or dropouts. Prot Def $=$ Protocol deficiencies. $\downarrow$ Power $=$ Insufficient statistical power. $\uparrow$ Side effcs $=$ Intervention had excessive side effects.

events. This table purposely does not focus on the studies in which a lack of a reduction in cardiovascular death was also cited as an outcome of interest. The reasons for this approach are multiple. First, none of the cited studies considered only death as the primary outcome and therefore none were statistically powered to detect cardiovascular death. Second, major improvements (e.g., beta blockers, angiotensin receptor blockers, anti-arrythmics, etc.) employed in the treatment of acute coronary syndromes have occurred in the last decade which greatly increase the survival of patients with a myocardial infarction or stroke [13]. These advances greatly reduce mortality unrelated to the study's primary intervention. Third, death from a myocardial infarction (e.g., congestive heart failure) may occur many years after a heart attack, much longer than the limited observation time of most studies.

\section{Protocol Deficiencies}

The following limitations in study design, study execution, or study complications may have greatly limited the ability to assess the effect of LDLc lowering on 
the prevention of cardiovascular events. Examination of each of the listed studies considers whether one or more of the following limitations apply (Table 1).

1) Too short an observation time ( $\downarrow$ Obser). The significant reduction of cardiovascular events requires time, sometimes as long as seven years or more as shown in the IMPROVE-IT clinical trial [14]. The length of time depends on many variables including the percentage of LDLc lowering, the magnitude of the cardiovascular risk of the study cohort, and the concomitant change in C-reactive protein (CRP). Short-term studies may not allow enough time to accumulate sufficient events to reach statistical significance.

2) Failure to monitor or normalize the concomitant change in C-reactive protein (CRP?). Randomized Controlled Clinical Trials have demonstrated that significant lowering of CRP is as important as lowering LDLc in preventing cardiovascular events [15]. If CRP does not normalize or minimally decreases, minimal effects of LDLc lowering would be expected. Therefore, it is critical to monitor this inflammatory marker for normalization $(<1.0 \mathrm{mg} / \mathrm{L})$ before a negative study outcome should be considered.

3) Use of an LDLc lowering agent that does not increase hepatic LDL receptors (LDL Rcpt). Recent studies have emphasized the importance of increasing hepatic LDL receptors as the principle mechanism of lowering plasma LDLc in order to reduce cardiovascular events [16]. Hepatic removal of LDLc is the primary method for secreting cholesterol into the bile/gut with its eventual elimination in the stool. Other methods of lowering LDLc (such as clearance by macrophages) would not be expected to lower the tissue content of cholesterol-rich atherosclerotic plaques. Substances or activities that increase the hepatic LDL receptor are low calorie diet, bile acid sequestrants, ileal bypass surgery, statins, ezetimibe, PCSK9 inhibitors, and estrogens. Substances or activities that lower LDL cholesterol but do not increase hepatic LDL receptors include fibrates, cholesterol ester transfer protein inhibitors, and niacin [16] [17]. Therefore, it is important in evaluating an LDL reduction study to be certain that the intervention that was used increases hepatic LDL receptors.

4) Unexpectedly low rate of cardiovascular events ( $\downarrow$ CVD). The power to detect a reduction in cardiovascular events is dependent on the number of expected events that occur during the study. In many studies, a smaller number of events occurred than are expected in the control group. The reasons for this are complex and include the fact that participants in clinical trials have better outcomes than the non-studied general population. This has been termed the "Hawthorne Effect" [18]. When the number of anticipated outcome events is reduced, the power of the study to detect a significant difference is also reduced.

5) Lack of reaching the significant level of $\mathrm{p}<0.05$ ( $\downarrow$ Stat). A study may show a numerical benefit but not a statistical difference in outcome. The traditional use of $\mathrm{p}<0.05$ is a very conservative statistic, designed to avoid false positive conclusions. We approve of this approach, but it may often lead to the wrong conclusion, i.e., that the study is demonstrating no beneficial effect of LDLc lowering (a false negative/type 2 statistical error) when a positive result is 
the true outcome. This is the reason that a negative study conclusion should be viewed with extreme caution [19].

6) Excessive dropins or dropouts ( $\uparrow$ Drop in/out). It is not uncommon for a new therapeutic modality to become available during the duration of any trial. For example, during clinical trials involving simvastatin, atorvastatin became available. Atorvastatin is more potent than simvastatin and is associated with less myopathy, particularly at maximal dosages. When study participants ingest an alternative therapeutic agent, this change is termed "dropin." Dropin numbers are very difficult to predict when studies are designed. The design of every study which uses an "intention to treat" analysis includes an allowance for individuals to crossover to the alternative arm of the study (crossovers) as well as participants who stop participating in the trial (dropouts). When this occurs, the power of the study to detect a difference is compromised [20]. Both excessive dropins and dropouts can prevent a clinical trial from identifying a significant difference in outcome between study arms.

7) Protocol deficiencies (Prot Def). Most protocols designs are based upon data obtained from previous small, non-randomized studies. They are designed by a committee of experts in various fields and are therefore valid for the specific question being addressed. During the recruitment and operation of the protocol, additional information may become available which invalidates the original experimental design. This information is often detrimental for making study conclusions.

8) Lack of Statistical Power ( $\downarrow$ Power). In almost all randomized, controlled studies, the power analysis calculation indicates the number of individuals who must be included in the study and the duration of the study. It is determined by the primary question of the study. Asking secondary questions, even when prespecified, is rarely sufficiently powered to provide answers with confidence. Secondary analysis should only be considered exploratory, not definitive.

9) Intervention Side Effects ( $\uparrow$ Side effcs). It is not uncommon for a new therapeutic agent to have untoward side effects that counteract the potential benefits of the intervention. Common side effects include hypertension, nausea and vomiting, palpitations, anxiety, etc. Side effects often encourage the participant to reduce compliance with the intervention medication or drop out from the clinical trial. Excessive side effects may obfuscate the true outcome that the primary intervention has a significant beneficial effect.

There are no perfect protocols because of limitations on funding, available populations, resources, and duration. Many conditions change after the initiation of any study which may invalidate the results. However, if a statistically significant primary end point is observed in an intention to treat, randomized, blinded study, the result is usually valid. In contrast, when a negative result is observed, great caution must be exercised is accepting the negative result as valid. The reason is that many unforeseen obstacles in study design and execution may be the cause for the negative result. All the articles cited in support of the tenet that lowering LDLc does not reduce CVD events are replete with major 
Table 2. Comments and concerns unique to each study.

\#1 ST. FRANCIS—authors state "study was underpowered and undertreated".

\#2 IDEAL-not blinded and better adherence to control than atorvastatin intervention.

\#3 FIELD-More placebo subjects added confounding statin therapy $(\mathrm{p}<0.001)$.

\#4 4D-Atorvastatin reduced the rate of all cardiovascular events combined $(\mathrm{p}<0.03)$.

\#5 ASPEN-Protocol changed at 2 years from secondary to primary intervention.

\#6 WHI-Only $8.6 \%$ of the participants had lipid profiles measured.

\#7 ILLUMINATE-Trial terminated early due adverse effects of the CETP inhibitor.

\#8 CDRONA-Heart failure only patients, usually excluded from CVD trials.

\#9 SEAS-Simvastatin plus ezetimibe reduced all ischemic CVD events $(\mathrm{p}<0.02)$.

\#10 GISSI-HF-Study examined death from heart failure, irrespective of cause.

\#11 AURORA-The extremely high CRP indicating inflammation was never reduced.

\#12 SEARCH-Excessive myopathy in the simvastatin arm vs. control (53 vs. 2).

\#13 AIM-HIGH-In a niacin trial, all patients also received simvastatin plus ezetimibe.

\#14 SDHS-Critical data lost, no LDLc data reported. Protocol out of date.

\#15 HPS2-THRIVE-Niacin caused a significant increase in diabetes, bleeding, etc.

\#16 ACCELERATE-The CETP inhibitor raised blood pressure \& C-reactive protein.

\#17 HIJ-PROPER-Ezetimibe significantly reduced CVD events in hyper-absorbers.

\#18 EMPATHY-Japanese study which lost patients and data during an earth quake.

Most of the above unanticipated flaws occurred in the execution and follow-up of the study and were not included in the initial design of the study protocol.

deficiencies that can invalidate a positive finding (Table 1 and Table 2). Table 1 describes the identified protocol shortcomings. Table 2 briefly lists concerns and comments that are unique to the execution of each study. In contrast to these studies, there are numerous well-designed studies that reach the statistically significant conclusion that lowering LDLc does reduce CVD events [7] [8] [9]. From the above analysis, it is evident that many of the cited clinical trials purportedly suggesting that lowing LDLc is not effective in reducing cardiovascular events are complicated by defective protocols, unforeseen events, or limited clinical trial data. This is the reason that the methodology and results of each clinical trial need to be carefully scrutinized.

\section{Conclusion}

Our analysis of the limitations of the clinical trials purporting to negate the LDL hypothesis strongly suggests that the cited trials have major deficiencies in design, methodology, or execution. In contrast, there is overwhelming randomized, blinded clinical trial data in the last decade strongly supporting an essential role for LDLc in the pathogenesis of atherosclerosis [10]. However, it needs to be recognized that LDLc is not the only pathogenic factor. Inflammation is an equally important contributing factor and is very important in the process of 
buildup of a fatty streak and atherosclerotic plaque development. Studies examining the effects of intervention with pharmaceuticals that are designed to lower cardiovascular events need to consider both LDLc changes as well as inflammatory alterations in the coronary arteries.

\section{Conflicts of Interest}

The authors declare no conflicts of interest regarding the publication of this paper.

\section{References}

[1] Hansson, G.K. (2005) Inflammation, Atherosclerosis, and Coronary Artery Disease. The New England Journal of Medicine, 352, 1685-1695.

https://doi.org/10.1056/NEJMra043430

[2] Schade, D.S., Murphy, S., Exil, V. and Eaton, P.R. (2018) A Pediatric Opportunity in Adolescents to Prevent Adult Heart Attacks. World Journal of Cardiovascular Diseases, 8, 85-101. https://doi.org/10.4236/wjcd.2018.82009

[3] Libby, P. (2013) Mechanisms of Acute Coronary Syndromes and Their Implications for Therapy. The New England Journal of Medicine, 368, 2004-2013. https://doi.org/10.1056/NEJMra1216063

[4] Libby, P. (2002) Atherosclerosis: The New View. Scientific American, 286, 46-55. https://doi.org/10.1038/scientificamerican0502-46

[5] Gao, W.-Q., Feng, Q.-Z., Li, Y.-F., Li, Y.-X., Huang, Y., Chen, Y.-M., et al. (2014) Systematic Study of the Effects of Lowering Low-Density Lipoprotein-Cholesterol on Regression of Coronary Atherosclerotic Plaques Using Intravascular Ultrasound. BMC Cardiovascular Disorders, 14, 60-78. https://doi.org/10.1186/1471-2261-14-60

[6] Ridker, P.M., Rifai, N., Pfeffer, M.A., Sacks, F.M., Moye, L.A., Goldman, S., et al. (1998) Inflammation, Pravastatin, and the Risk of Coronary Events after Myocardial Infarction in Patients with Average Cholesterol Levels. Circulation, 98, 839-844. https://doi.org/10.1161/01.CIR.98.9.839

[7] Baigent, C., Keech, A., Kearney, P.M., Blackwell, L., Buck, G., Pollicino, C., et al. (2005) Efficacy and Safety of Cholesterol-Lowering Treatment: Prospective Meta-Analysis of Data from 90,056 Participants in 14 Randomised Trials of Statins. The Lancet, 366, 1267-1278. https://doi.org/10.1016/S0140-6736(05)67394-1

[8] Cholesterol Treatment Trialists' (CTT) Collaboration (2010) Efficacy and Safety of more Intensive Lowering of LDL Cholesterol: A Meta-Analysis of Data from 170,000 Participants in 26 Randomised Trials. The Lancet, 376, 1670-1681. https://doi.org/10.1016/S0140-6736(10)61350-5

[9] Mihaylova, B., Emberson, J., Blackwell, L., Keech, A., Simes, J., Barnes, E.H., et al. (2012) The Effects of Lowering LDL Cholesterol with Statin Therapy in People at Low Risk of Vascular Disease: Meta-Analysis of Individual Data from 27 Randomised Trials. The Lancet, 380, 581-590.

[10] Schade, D.S., Cavanaugh, B., Ramo, B. and Eaton, R.P. (2016) The Application of the LDL Principle. World Journal of Cardiovascular Diseases, 6, 109-125. https://doi.org/10.4236/wjcd.2016.65012

[11] DuBroff, R. (2018) A Reappraisal of the Lipid Hypothesis. The American Journal of Medicine, 131, 993-997. https://doi.org/10.1016/j.amjmed.2018.04.027

[12] Nissen, S.E. (2017) Statin Denial: An Internet-Driven Cult with Deadly Conse- 
quences. Annals of Internal Medicine, 167, 281-282.

https://doi.org/10.7326/M17-1566

[13] Fox, K.A.A., Steg, P.G., Eagle, K.A., Goodman, S.G., Anderson, F.A., Granger, C.B., et al. (2007) Decline in Rates of Death and Heart Failure in Acute Coronary Syndromes, 1999-2006. The Journal of the American Medical Association, 297, 1892-1900. https://doi.org/10.1001/jama.297.17.1892

[14] Cannon, C.P., Blazing, M.A., Giugliano, R.P., McCagg, A., White, J.A., Theroux, P., et al. (2015) Ezetimibe Added to Statin Therapy after Acute Coronary Syndromes. The New England Journal of Medicine, 372, 2387-2397. https://doi.org/10.1056/NEJMoa1410489

[15] Ridker, P.M., Danielson, E., Fonseca, F.A.H., Genest, J., Gotto, A.M., Kastelein, J.J.P., et al. (2008) Rosuvastatin to Prevent Vascular Events in Men and Women with Elevated C-Reactive Protein. The New England Journal of Medicine, 359, 2195-2207. https://doi.org/10.1056/NEJMoa0807646

[16] Silverman, M.G., Ference, B.A., Im, K., Wiviott, S.D., Giugliano, R.P., Grundy, S.M., Braunwald, E. and Sabatine, M.S. (2016) Association between Lowering LDL-C and Cardiovascular Risk Reduction among Different Therapeutic Interventions: A Systematic Review and Meta-Analysis. The Journal of the American Medical Association, 316, 1289-1297. https://doi.org/10.1001/jama.2016.13985

[17] Parini, P., Angelin, B. and Rudling, M. (1997) Importance of Estrogen Receptors in Hepatic LDL Receptor Regulation. Arteriosclerosis, Thrombosis, and Vascular Biology, 17, 1800-1805. https://doi.org/10.1161/01.ATV.17.9.1800

[18] McCarney, R., Warner, J., Iliffe, S., van Haselen, R., Griffin, M. and Fisher, P. (2007) The Hawthorne Effect: A Randomized, Controlled Trial. BMC Medical Research Methodology, 7, 30-37. https://doi.org/10.1186/1471-2288-7-30

[19] Pocock, S.J. and Stone, G.W. (2016) The Primary Outcome Fails-What Next? The New England Journal of Medicine, 375, 861-870. https://doi.org/10.1056/NEJMra1510064

[20] McDonough, P.G. (2002) The Randomized World Is Not without Its Imperfections: Reflections on the Women's Health Initiative Study. Fertility and Sterility, 78, 951-956. https://doi.org/10.1016/S0015-0282(02)04403-5 